\title{
Rate Allocation for Wireless Multimedia Sensor Networks Using Pricing Mechanism
}

\author{
Zhang Jian Ming, ${ }^{1}$ Wang YI, ${ }^{1,2}$ Xie Ze Ming, ${ }^{2}$ Tang Shi Yi, ${ }^{2}$ and Ou Hao Yuan \\ ${ }^{1}$ School of Information Engineering, Guangdong Polytechnic Normal University, Guangdong 528041, China \\ ${ }^{2}$ School of Information and Engineering, South China University of Technology, Guangdong 510641, China \\ Correspondence should be addressed to Wang YI; wangy_gdptc@163.com
}

Received 9 October 2014; Accepted 24 December 2014

Academic Editor: Guangzhong Xie

Copyright (c) 2015 Zhang Jian Ming et al. This is an open access article distributed under the Creative Commons Attribution License, which permits unrestricted use, distribution, and reproduction in any medium, provided the original work is properly cited.

\begin{abstract}
A bandwidth allocation algorithm for wireless multimedia sensor networks is proposed in this paper. On the premise of Initial allocation algorithm based on distortion and congestion model to provide an efficient system performance, the allocation algorithm based on pricing mechanism we proposed balances the real-time requirement of different users by adjusting the bandwidth price in each network and each slot. Simulation results show that the proposed algorithm balances the bandwidth ratio of different users according to the video complexity, and it can provide a good system performance guaranteeing the fairness among the users.
\end{abstract}

\section{Introduction}

In recent years, the growing interest in the wireless sensor network (WSN) has resulted in thousands of peer-reviewed publications. Most of this research is concerned with scalar sensor networks that measure physical phenomena, such as temperature, pressure, humidity, or location of objects that can be conveyed through low-bandwidth and delaytolerant data streams. Recently, the focus is shifting toward research aimed at revisiting the sensor network paradigm to enable delivery of multimedia content, such as audio and video streams and still images, as well as scalar data [1]. WMSNs (wireless multimedia sensor networks) technology has emerged also due to the production of cheap CMOS (complementary metal oxide semiconductor) cameras and microphones, which can acquire rich media content from the environment like images and videos. WMSNs can be defined as networks of wirelessly interconnected sensor nodes equipped with multimedia devices, such as cameras that are capable of retrieving video and audio streams, images, and scalar sensor data [2].

Now the availability of complementary metal oxide semiconductor (CMOS) camera and small microphones makes the development of WMSNs capable of gathering the multimedia information from the surrounding environment possible. Its physical layer technologies can be classified based on the modulation scheme and bandwidth consideration [3] into three groups: narrow band, spread spectrum, and ultrawide band (UWB) technologies. Also they can be classified based on standard protocols into IEEE 802.15.4 ZigBee, IEEE 802.15.1 Bluetooth, IEEE 802.11 WiFi, IEEE 802.16 WiMAX, and 802.15.3a UWB.

Furthermore, WMSNs can perform online processing of the retrieved information and react to it by combining technologies from diverse disciplines such as wireless communications and networking, signal processing, computer vision, control, and robotics. There are mainstream algorithms coming from two theoretic methods: centralized method and decentralized method. In many cases, the algorithms are based on game theoretic model [4] (centralized method), where all of the users need to communicate their individual videos information to decide the bit-rate allocation at each slot. This information is so complicated which will put extra burdens on the networks. Instead of using game theoretic system, a number of algorithms based on optimum theory $[5,6]$ are proposed to improve the overall video quality. However the high overall quality comes at the expense of lowering the quality of some videos and may affect their realtime data transmission. 
Compared with previous algorithms [4-6], we present a multiuser video streaming rate allocation algorithm in wireless multimedia sensor networks based on supply and demand model [7] with pricing mechanism [8]. This algorithm only requires simpler information to be exchanged and does not require a heavy computational burden, whereas that burden will occur on a central controller. This scheme is modeled on price-guided procedures discussed in the economics literature that are characterized as decentralized, as various video users (senders) only communicate their bitrate demands in response to the bit-rate price announced by a bit-rate allocator in a slot. In our decentralized procedure, the allocator adjusts the user's demands to equalize the aggregate allocation to the available supply and announces the price for the next slot. With this price-guided allocation scheme, instead of using bits at a constant rate, users will increase their demand in slots during which their videos are more complex (e.g., high motion) and reduce their demand in slots of low complexity. Permitting the amount of bit rate used in each slot to vary increases the efficiency of each user's total bit rate by giving more of the resource when it is most valuable (in terms of lowering MSE (mean square error)) and less when it is less valuable. The use of a price to guide users' choices of demand reflects the relative scarcity of available bit rate in each network and each slot. When all users request more bits than the average, scarcity is greater and the price is higher, thus moderating the demands. Our simulation results show that each user benefits from this price-based decentralized bit-rate allocation mechanism compared with the equal bit-rate allocation to all users (absolute fairness in users). The performance of this algorithm is comparable to the centralized bit-rate allocation introduced in [9], where all users send their RD (rate/distortion) curves to the allocator. In the centralized approach, the computations grow exponentially with the number of users and the central controller is responsible for all of the computations. In the decentralized approach, the computational complexity remains constant for the allocator and is independent of the number of users. Each user makes the bit-rate demand calculations independently. The pricing-based method proposed in [8] is similar to ours but only considers that all multiuser video streams are sharing a common transmission channel, and it cannot be applied to heterogeneous networks for WMSNs.

The rest of the paper is organized as follows. Section 2 describes the initial allocation algorithm based on distortion and congestion model. Section 3 describes the proposed rate allocation algorithm and its process. Simulation results are given in Section 4, and Section 5 concludes the paper.

\section{Initial Allocation Algorithm}

In this section, we first briefly describe the network model and rate distortion model and then provide the initial allocation algorithm in detail.

Figure 1 shows a topology of heterogeneous network simulation, supposing that there are $I$ users sharing the available bandwidth in $N$ networks. The video stream is

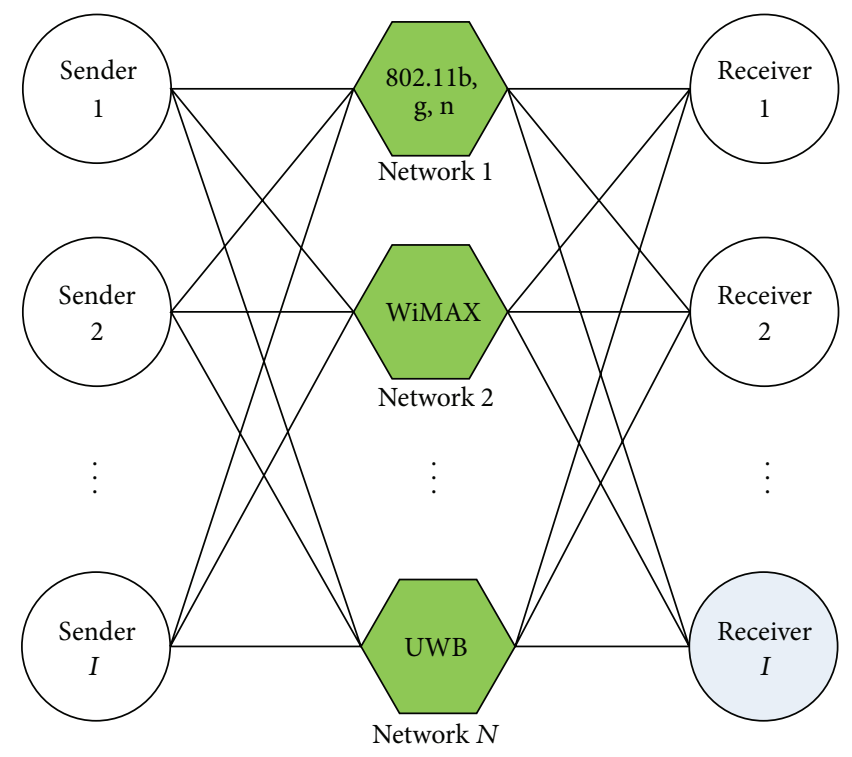

FIGURE 1: Topology for heterogeneous network simulations.

divided into time slots. For user $i$, let $R_{i, n}$ denote its bit rate in network $n$ and $R_{i}^{U}$ represent the total bit rate of user $i$ :

$$
R_{i}^{U}=\sum_{n=1}^{N} R_{i, n}, \quad i=1,2 \cdots I
$$

As for network $n$, the total bit rate of all users is

$$
R_{n}^{N}=\sum_{i=1}^{I} R_{i, n}, \quad n=1,2 \cdots N .
$$

Let $C_{n}$ be the available bandwidth of network $n$ and $e_{n}$ the remaining bandwidth. Their relations can be expressed as

$$
e_{n}=C_{n}-\sum_{i=1}^{I} R_{i, n}=C_{n}-R_{n}^{N}, \quad n=1,2 \cdots N .
$$

On the basis of above, we denote the congestion by the traffic flows over the network. Following the M/M/1 queuing model, the network congestion can be expressed as

$$
X\left(R_{i, n}\right)=\sum_{n=1}^{N} \frac{\sum_{i=1}^{I} R_{i, n}}{C_{n}-\sum_{i=1}^{I} R_{i, n}} .
$$

Let $D\left(R_{i}^{U}\right)$ denote the MSE distortion at a rate $R_{i}^{U}$. As given in [10], the $\mathrm{RD}$ curve can be fitted by

$$
D\left(R_{i}^{U}\right)=a_{i}+\frac{b_{i}}{R_{i}^{U}+d_{i}},
$$

where the parameters $a_{i}, b_{i}$, and $d_{i}$ are the coefficients of user $i$ which depend on the encoding strategy and INTRA rate $\beta_{i}$ [5]; they can be estimated by the nonlinear regression techniques. This model is widely used for $\mathrm{RD}$ curve fitting.

As we know, the average latency of the network $n$ is related to its allocated rate $R_{n}^{N}$; increasing $R_{n}^{N}$ would contribute to 
the network congestion. Meanwhile, decreasing the allocated rate would lower the congestion, but the videos would suffer extra distortion during encoding. Considering the two objectives above and the constraint of maximum rate $R_{i}^{\max }$ and minimum $R_{i}^{\mathrm{min}}$, the optimization problem can be expressed as

$$
\begin{array}{ll}
\min _{\left\{R_{i}^{U}, R_{i, n}\right\}} & \left\{\sum_{i=1}^{I}\left(a_{i}+\frac{b_{i}}{R_{i}^{U}+d_{i}}\right)+k \sum_{n=1}^{N} \frac{\sum_{i=1}^{I} R_{i, n}}{C_{n}-\sum_{i=1}^{I} R_{i, n}}\right\} \\
\text { s.t. } \quad & R_{i}^{\min } \leq R_{i}^{U} \leq R_{i}^{\max }, \quad i=1,2 \cdots I \\
& R_{n}^{N} \leq C_{n}, \quad n=1,2 \cdots N,
\end{array}
$$

where $k$ is the weighting factor of congestion and distortion.

Allowing for the fairness of allocated bandwidth in different network, the relation between $R_{i, n}$ and $R_{i}^{U}$ can be expressed as

$$
R_{i, n}=\rho_{i, n} R_{i}^{U}, \quad n=1,2 \cdots N .
$$

In (9), the allocation ratio parameter $\rho_{i, n}$ is a design choice to make sure that the allocated bandwidth is proportional to the observed bandwidth. As given in [5], following the weighted proportional fairness in different networks, $\rho_{i, n}$ can be calculated as

$$
\rho_{i, n}=\frac{C_{i, n}}{\sum_{n=1}^{N} C_{i, n}}=\frac{C_{n}}{\sum_{n=1}^{N} C_{n}}=\rho_{n},
$$

where $\rho_{n}$ is the proportion of $C_{n}$ in the total available bandwidth.

Using (4), then (9) can be derived as

$$
R_{i, n}=\rho_{i, n} R_{i}^{U}=\rho_{n} R_{i}^{U}
$$

Using (11), (8) can be expressed as

$$
\sum_{i=1}^{I} R_{i}^{U} \leq \sum_{n=1}^{N} C_{n}
$$

Using (11) in (9), then the optimization problem becomes

$$
\begin{array}{r}
\min _{\left\{R_{i}^{U}\right\}}\left\{\sum_{i=1}^{I}\left(a_{i}+\frac{b_{i}}{R_{i}^{U}+d_{i}}\right)+k \sum_{n=1}^{N} \frac{\sum_{i=1}^{I} R_{i}^{U}}{\sum_{n=1}^{N} C_{n}-\sum_{i=1}^{I} R_{i}^{U}}\right\} \\
i=1,2 \cdots I, \quad n=1,2 \cdots N
\end{array}
$$

subject to the constraint

$$
\begin{gathered}
R_{i}^{\min } \leq R_{i}^{U} \leq R_{i}^{\max }, \quad i=1,2 \cdots I, \\
\sum_{i=1}^{I} R_{i} \leq \sum_{n=1}^{N} C_{n}, \quad i=1,2 \cdots I, n=1,2 \cdots N .
\end{gathered}
$$

As given in [11], we adopt the parallel hybrid recollection algorithm to calculate the initial allocated bandwidth.

\section{Pricing Mechanism Allocation Algorithm}

At time slot $t$, we set the transfer rate of user $i$ as $R_{i}^{U}(t)$. Denote $U_{i, t}\left(R_{i}^{U}(t)\right)$ to be the utility of user $i$ at the rate of $R_{i}^{U}(t)$. As given in [4], it can be expressed as the negative of the MSE distortion:

$$
U_{i, t}\left(R_{i}^{U}(t)\right)=-D\left(R_{i}^{U}(t)\right)=-\left(a_{i, t}+\frac{b_{i, t}}{R_{i}^{U}(t)+d_{i, t}}\right) .
$$

We set per unit of bandwidth a price, and it will fluctuate according to the supply and demand of bandwidth. At each slot, the transmitted video stream will spend an amount of wealth based on the allocated bandwidth and current bandwidth price. Before entering the system, the users will be allocated an initial wealth based on the length of the video and the initial allocated bandwidth. Therefore, as for the user, the optimization problem for the real-time rate allocation is to maximize his utility while the expended wealth over all slots is no more than the initial wealth.

For user $i$, the transmission starts at slot $T_{S}$ and ends at slot $T_{E}$. Therefore, the initial wealth $W_{i, T_{S}}$ can be expressed as

$$
W_{i, T_{S}}=\left(T_{E}-T_{S}+1\right) * R_{i}^{U}\left(T_{S}\right) * \bar{p}_{T_{S}}
$$

where $\bar{p}_{T_{S}}$ is the initial bandwidth price and normalizes as $\bar{p}_{T_{S}}=1$ and $R_{i}^{U}\left(T_{S}\right)$ is the initial allocation bandwidth.

Consider the optimization objective and the utility model as given, at slot $t_{m}$, within the limitations of remaining wealth $W_{i, t_{m}}$; when the rate of user $i$ reaches his maximum, his utility goes to the maximum at the same point:

$$
\begin{array}{ll}
\max _{\left\{R_{i}^{U}(t)\right\}} & \sum_{t=t_{m}}^{T_{E}} U_{i, t}\left(R_{i}^{U}(t)\right) \\
\text { s.t. } & \sum_{t=t_{m}}^{T_{E}} p_{t} * R_{i}^{U}(t) \leq W_{i, t_{m}},
\end{array}
$$

where $p_{t}$ represents the bandwidth price of slot $t_{m}$.

However, in the real-time transmission, the coefficients for overall future slots are unknown. Thus, we divide the overall waiting slots into two parts, the current slot and the future slots, and the optimization problem becomes

$$
\begin{array}{ll}
\max _{\substack{R_{i, t_{m}}^{\text {req }}, \bar{R}_{i . t_{m}}^{\text {req }}\\
}}\left\{-\left(a_{i, t_{m}}+\frac{b_{i, t_{m}}}{\left(R_{i, t_{m}}^{\text {req }}+d_{i, t_{m}}\right)}\right)\right. \\
& \left.-\left(T_{E}-t_{m}\right) *\left(\bar{a}_{i, t_{m}}+\frac{\bar{b}_{i, t_{m}}}{\left(\bar{R}_{i, t_{m}}^{\mathrm{req}}+\bar{d}_{i, t_{m}}\right)}\right)\right\} \\
\text { s.t. } & p_{t_{m}} * R_{i, t_{m}}^{\mathrm{req}}+\left(T_{E}-t_{m}\right) * \bar{p}_{t_{m}} * \bar{R}_{i, t_{m}}^{\mathrm{req}} \leq W_{i, t_{m}},
\end{array}
$$

where $\bar{a}_{i, t_{m}}, \bar{b}_{i, t_{m}}$, and $\bar{d}_{i, t_{m}}$ are the future average coefficients and $\bar{p}_{t_{m}}$ represents the future average bandwidth price. 
Suppose that the future slots are stable and predictable; then the future average coefficients can be estimated by a number of slots stored in the buffer. As given in [11], considering the convex-concave feature, we use Lagrange multiplier approach to calculate the request bandwidth as follows:

$$
\begin{aligned}
R_{i, t_{m}}^{\mathrm{req}}= & \sqrt{\frac{b_{i, t_{m}}}{p_{t_{m}}}} \\
& +\frac{W_{i, t_{m}}+p_{t_{m}} * d_{i, t_{m}}+\left(T_{E}-t_{m}\right) * \bar{p}_{t_{m}} * \bar{d}_{i, t_{m}}}{\sqrt{p_{t_{m}} * b_{i, t_{m}}}+\left(T_{E}-t_{m}\right) * \sqrt{\bar{p}_{t_{m}} * \bar{b}_{i, t_{m}}}} \\
& -d_{i, t_{m}}, \quad i=1,2 \cdots I .
\end{aligned}
$$

As the stationary of video stream, we can set $\bar{p}_{t_{m}}=1$.

Since the request bandwidth of all users will not be equal to the available bandwidth, the central controller will normalize the total request bandwidth to allocate available bandwidth as the supply and demand

$$
R_{i}^{U}\left(t_{m}\right)=\sum_{n=1}^{N} C_{n, t_{m}} * \frac{R_{i, t_{m}}^{\mathrm{req}}}{\sum_{i=1}^{I} R_{i, t_{m}}^{\mathrm{req}}}
$$

and adjust the bandwidth price of the next slot:

$$
p_{t_{m}+1}=p_{t_{m}}+\mu_{p}\left(\frac{\sum_{i=1}^{I} R_{i, t_{m}}^{\mathrm{req}}-\sum_{n=1}^{N} C_{n, t_{m}}}{\sum_{n=1}^{N} C_{n, t_{m}}}\right) .
$$

Users encode the video streams according to the allocated bandwidth $R_{i}^{U}\left(t_{m}\right)$ and transmit through different networks as the allocation ratio parameter $\rho_{i, n}$ :

$$
R_{i, t_{m}}^{n}=\rho_{i, n} R_{i}^{U}\left(t_{m}\right)
$$

Then, update the remaining wealth of slot $t_{m+1}$ by the cost:

$$
W_{i, t_{m}+1}=W_{i, t_{m}}-p_{t_{m}} * R_{i}^{U}\left(t_{m}\right) .
$$

Here, we will represent the algorithm flow.

Step 1 (initialization). According to the coefficients of different video streams, the central controller calculates the initial allocated bandwidth by using (13), and by (16), each user calculates his initial wealth separately.

Step 2 (request bandwidth calculation). At slot $t_{m}$, each user calculates his request bandwidth by (19) and transfers to the central controller.

Step 3 (bandwidth allocation and price adjustment). According to the request bandwidth ratio of different users, central controller calculates the allocated bandwidth by (20) and uses (21) to adjust the bandwidth price of slot $t_{m+1}$.

Step 4 (transmission and remaining wealth updating). Using (22), users allocate the bit rate to different networks and update their remaining wealth of slot $t_{m+1}$ by using (23).
TABLE 1: Video coefficients of three users.

\begin{tabular}{cccc}
\hline & $\beta_{i}$ & $R_{i}^{\min }$ & $R_{i}^{\max }$ \\
\hline$U 1$ & 0.0409 & $0.4998 \mathrm{Mbps}$ & $7.1134 \mathrm{Mbps}$ \\
U2 & 0.0025 & $3.0692 \mathrm{Mbps}$ & $22.3412 \mathrm{Mbps}$ \\
U3 & 0.0021 & $2.4581 \mathrm{Mbps}$ & $15.0274 \mathrm{Mbps}$ \\
\hline
\end{tabular}

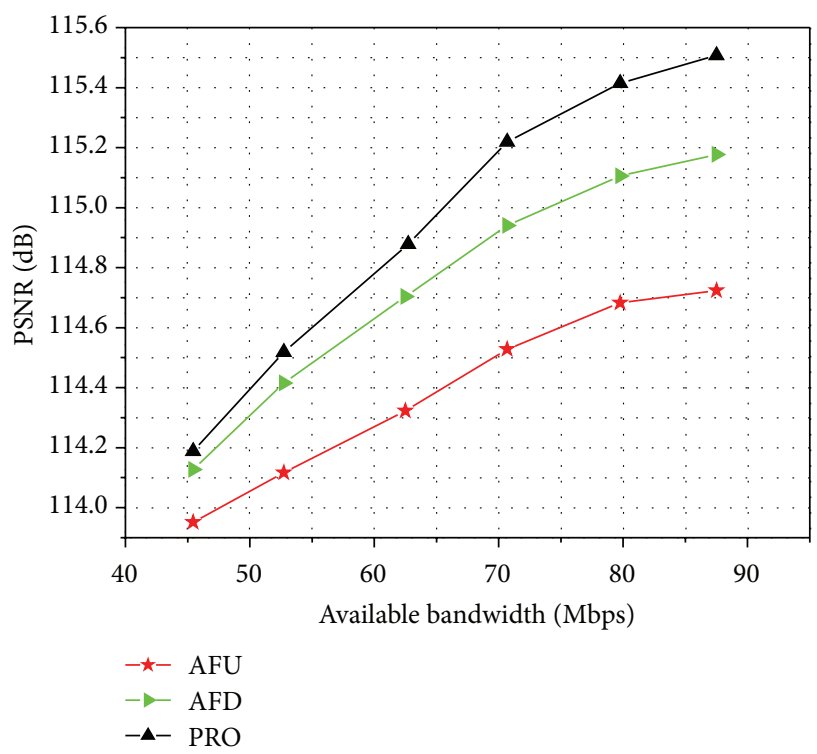

FIGURE 2: PSNR comparison under 6 available bandwidth scenarios.

TABLE 2: Available bandwidth scenarios.

\begin{tabular}{ccccc}
\hline & $C_{1}$ & $C_{2}$ & \multicolumn{1}{c}{$C_{3}$} & $C_{1}+C_{2}+C_{3}$ \\
\hline 1 & $21.2 \mathrm{Mbps}$ & $10.5 \mathrm{Mbps}$ & $3.2 \mathrm{Mbps}$ & $45.6 \mathrm{Mbps}$ \\
2 & $24.7 \mathrm{Mbps}$ & $11.9 \mathrm{Mbps}$ & $3.5 \mathrm{Mbps}$ & $52.9 \mathrm{Mbps}$ \\
3 & $29.4 \mathrm{Mbps}$ & $14.1 \mathrm{Mbps}$ & $3.9 \mathrm{Mbps}$ & $62.7 \mathrm{Mbps}$ \\
4 & $33.1 \mathrm{Mbps}$ & $15.9 \mathrm{Mbps}$ & $4.5 \mathrm{Mbps}$ & $70.7 \mathrm{Mbps}$ \\
5 & $37.1 \mathrm{Mbps}$ & $17.7 \mathrm{Mbps}$ & $5.6 \mathrm{Mbps}$ & $79.8 \mathrm{Mbps}$ \\
6 & $40.9 \mathrm{Mbps}$ & $19.7 \mathrm{Mbps}$ & $5.8 \mathrm{Mbps}$ & $87.6 \mathrm{Mbps}$ \\
\hline
\end{tabular}

Step 5 (judgment). Repeat Step 2 to Step 4 until all videos are transferred.

\section{Simulation Results}

Simulation results are performed in a heterogeneous network which consists of three different multimedia sensor networks. The number of users is three and the coefficients of the videos are shown in Table 1.

To start with, we chose 6 increasing available bandwidth scenarios (Table 2) to simulate the initial allocation algorithm and compare with absolute-fairness-among-users (AFU) and absolute-fairness-in-distortion (AFD) algorithms.

In Figure 2, we compare the PSNR (peak signal-to-noise ratio) of the three algorithms. It can be noticed that the initial allocation leads to much higher PSNR compared with the other algorithms. This is because the initial allocation 


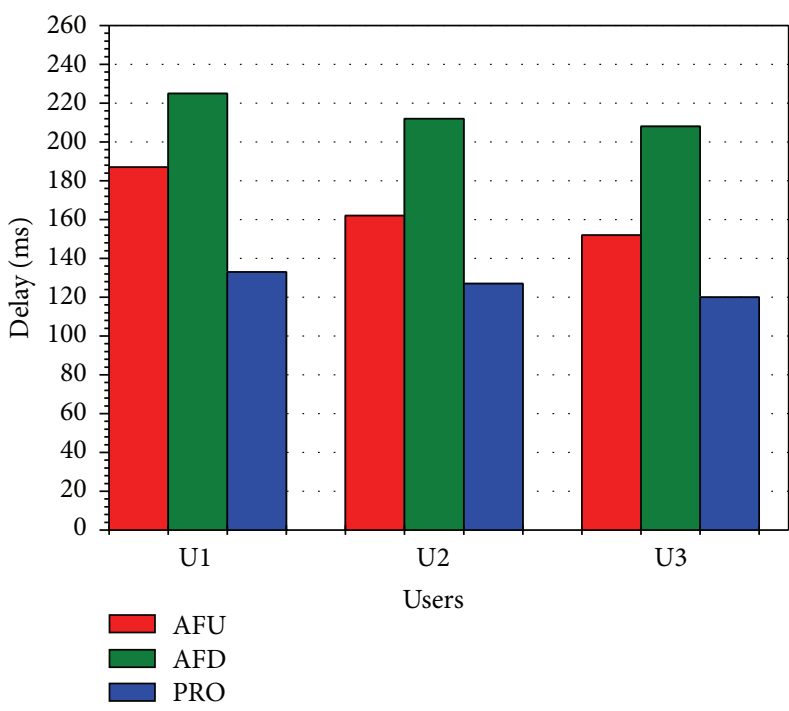

(a) Scenario $1\left\{C_{1}=21.2 \mathrm{Mbps}, C_{2}=10.5 \mathrm{Mbps}, C_{3}=3.2 \mathrm{Mbps}\right\}$

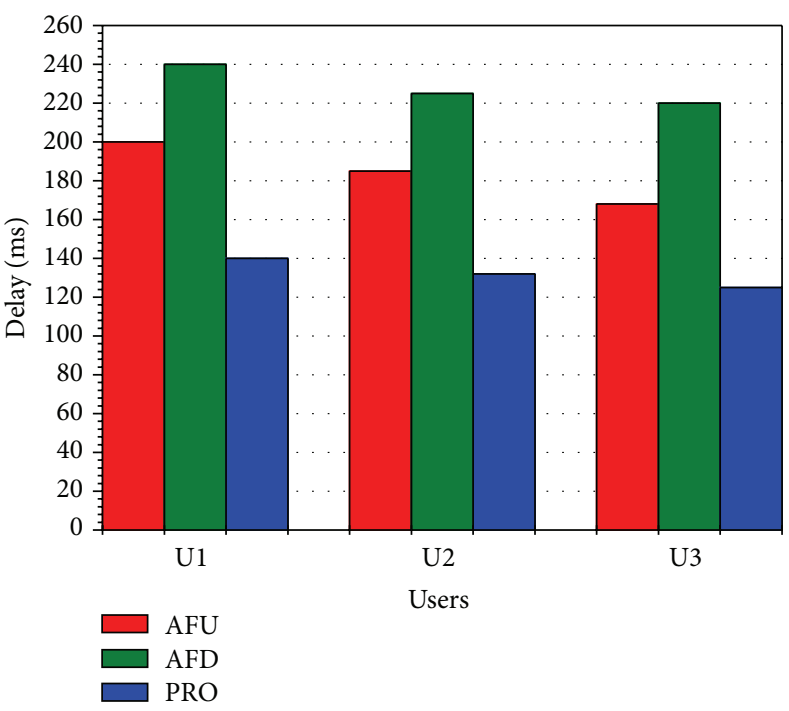

(b) Scenario $4\left\{C_{1}=33.1 \mathrm{Mbps}, C_{2}=15.9 \mathrm{Mbps}, C_{3}=4.5 \mathrm{Mbps}\right\}$

FIgURE 3: Average delivery delay comparison under scenarios 1 and 4.

considers the distortion of all video streams and seeks to improve the overall performance of PSNR. The AFU treats the flows with equal importance and takes no consideration of the source characteristics, which may lead to the unused bandwidth in simple videos and the increased distortion in complex videos due to the insufficient bit rate. Under the smaller bandwidth scenarios, the AFD performs close to the initial allocation. However, as the bandwidth is increasing, the gap is widening. This is because AFU just considers the absolute distortion rate and takes no account of the overall performance, which would have a limited improvement when bandwidth increases.

Figure 3 compares the average delivery delay under scenario 1 and scenario 4. In AFU and AFD algorithms, the average delivery delay would be higher; that is because allocated bit rates are reduced only after the congestion has been detected, and the congestion is lasting for a while. The initial allocation algorithm, on the other hand, considers the network latency and tries to avoid overcongestion by using a proactive strategy in the optimizing model, which significantly lower the average delay.

In the second stage, we simulate the real-time allocation algorithm under the same environment and compare it to AFU and media-aware algorithm [6]. As given in [12], we chose the FORWARD and BACKWARD prediction to estimate the future coefficients and calculate the request bandwidth.

In Figure 4, we notice that the overall PSNR of mediaaware algorithm and our algorithm are very close and much higher than AFU. That is, both of the two methods consider the overall MSE distortion and provide good overall performance.

As for the users, we compare their PSNR separately in Figure 5; it is shown that, as for the simple videos, the proposed allocation algorithm gets a little bit lower PSNR than media-aware algorithm does, while being significantly higher

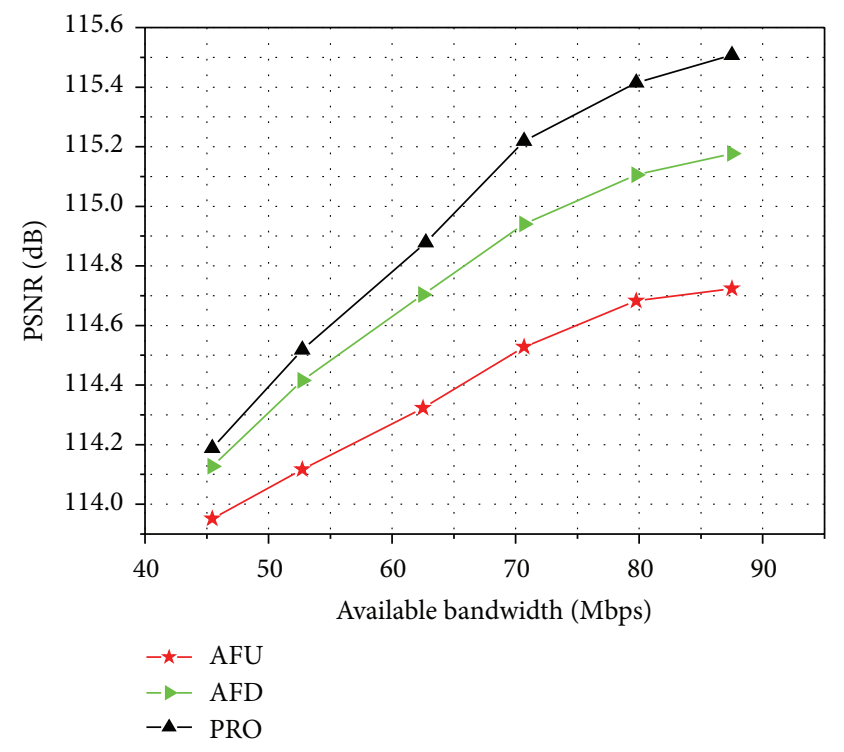

FIGURE 4: PSNR comparison under 6 available bandwidth scenarios.

for the complex videos. It is because the objective of mediaaware method is to allocate the bandwidth prior to simple videos and to get the maximum of the PSNR. It does better in improving the total PSNR than our method; however, not all users improve their video quality, especially on the condition of small bandwidth; some complex videos suffer worse PSNR compared with AFU (as shown in Figure 5(c)). As given in [13], this allocated result would have no improvement in video quality and make an impact on the normal use. In contrast, our method balances the bandwidth ratio between simple video and complex video; so all users are better than AFU and guarantee their video qualities. In general, under the similar total PSNR conditions, considering the 


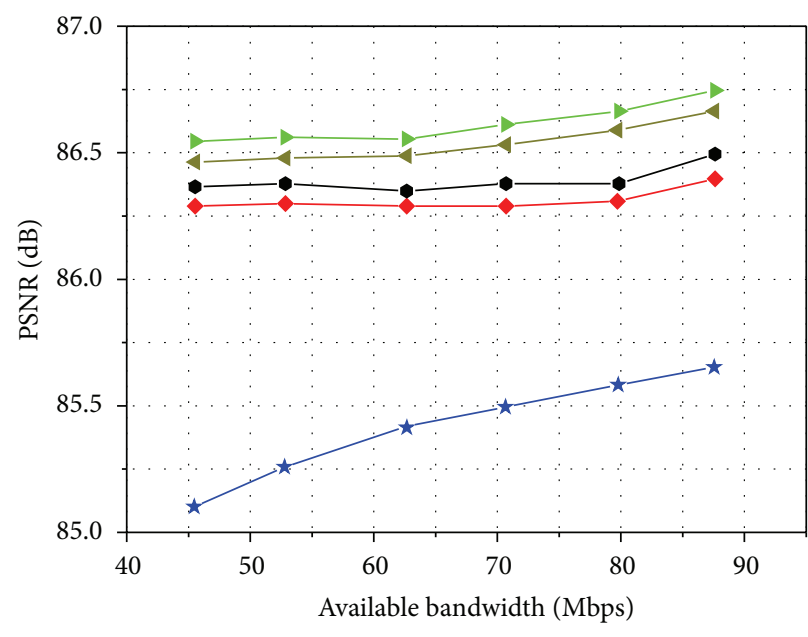

(a) PSNR comparison of user 1

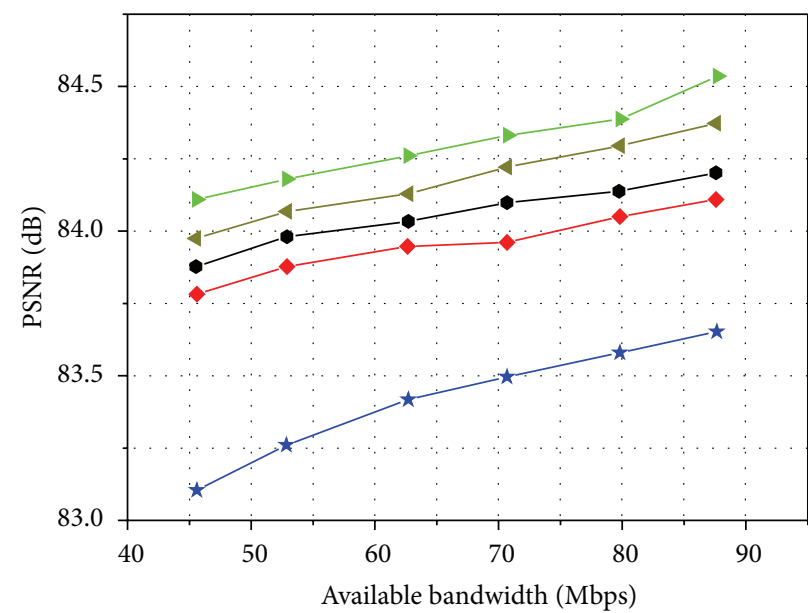

(b) PSNR comparison of user 2

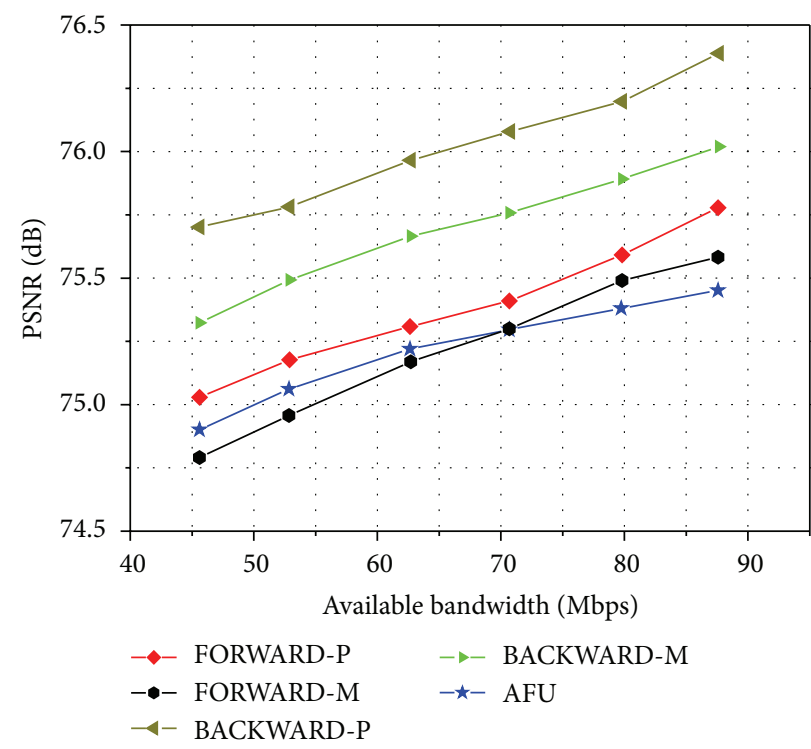

(c) PSNR comparison of user 3

FIGURE 5: PSNR comparison for different users under 6 available bandwidth scenarios.

fairness among users, our proposed method guarantees all users improve their quality, which is better than media-aware algorithm.

\section{Conclusion}

In this paper, considering the overall video performance in wireless multimedia sensor networks and the fairness among the users, we have proposed a bit-rate allocation algorithm for multiuser video streaming based on pricing mechanism.

As the simulation results show, on the premise of initial allocation algorithm based on distortion and congestion model to provide a good overall video performance and money limitation, the real-time allocation algorithm proposed utilizes the fluctuation of bandwidth price to adjust the rate of different users, which balances the bandwidth ratio of different users in different networks according to the video complexity. In comparison with existing methods [6], our method improves the overall system performance and guarantees the individual video quality among the users, whereas previous methods, focusing on improving the overall quality only, make some users improve at the expense of others.

\section{Conflict of Interests}

The authors declare that there is no conflict of interests regarding the publication of this paper.

\section{Acknowledgment}

This paper is supported by the National Natural Science Foundation of China (60971052 and 61103038). 


\section{References}

[1] I. F. Akyildiz, T. Melodia, and K. R. Chowdhury, "A survey on wireless multimedia sensor networks," Computer Networks, vol. 51, no. 4, pp. 921-960, 2007.

[2] W. Wolf, B. Ozer, and T. Lv, "Smart cameras as embedded systems," Computer, vol. 35, no. 9, pp. 48-53, 2002.

[3] K. D. Wong, "Physical layer considerations for wireless sensor networks," in Proceedings of the IEEE International Conference on Networking, Sensing and Control, vol. 2, pp. 1201-1206, Taipei, Taiwan, March 2004.

[4] Y. Chen, B. Wang, and K. J. R. Liu, "A game-theoretic framework for multi-user multimedia rate allocation," in Proceedings of the IEEE International Conference on Acoustics, Speech and Signal Processing (ICASSP '09), pp. 1997-2000, 2009.

[5] N. Lu and J. Bigham, "An optimal bandwidth adaptation algorithm for multi-class traffic in wireless networks," in Proceedings of the 3rd International Conference on Quality of Service in Heterogeneous Wired/Wireless Networks (QShine '06), ACM, September 2006.

[6] X. Zhu, P. Agrawal, J. P. Singh, T. Alpcan, and B. Girod, "Rate allocation for multi-user video streaming over heterogenous access networks," in Proceedings of the ACM 15th International Conference on Multimedia (MULTIMEDIA '07), pp. 37-46, 2007.

[7] Y. Chen, B. Wang, and K. J. Ray Liu, "Multiuser rate allocation games for multimedia communications," IEEE Transactions on Multimedia, vol. 11, no. 6, pp. 1170-1181, 2009.

[8] M. Tiwari, T. Groves, and P. Cosman, "Bit-rate allocation for multiple video streams using a pricing-based mechanism," IEEE Transactions on Image Processing, vol. 20, no. 11, pp. 3219-3230, 2011.

[9] M. Tiwari, T. Groves, and P. C. Cosman, "Competitive equilibrium bitrate allocation for multiple video streams," IEEE Transactions on Image Processing, vol. 19, no. 4, pp. 1009-1021, 2010.

[10] K. Stuhlmüller, N. Färber, M. Link, and B. Girod, "Analysis of video transmission over lossy channels," IEEE Journal on Selected Areas in Communications, vol. 18, no. 6, pp. 1012-1032, 2000.

[11] S. Boyd and L. Vandenberghe, Convex Optimization, Cambridge University Press, Cambridge, UK, 2004.

[12] I. Richardson, "H.264/MPEG-4 Part 10 White Paper: Intra Prediction[DB/OL]," May 2003, http://www.vcodex.com.

[13] X. Zhu, P. Agrawal, J. P. Singh, T. Alpcan, and B. Girod, "Distributed rate allocation policies for multihomed video streaming over heterogeneous access networks," IEEE Transactions on Multimedia, vol. 11, no. 4, pp. 752-764, 2009. 

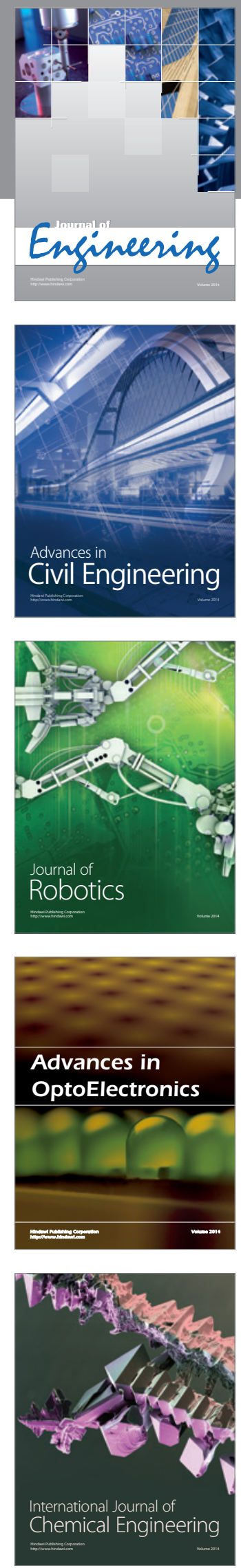

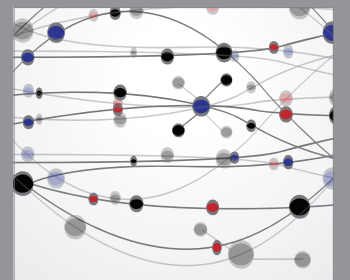

The Scientific World Journal
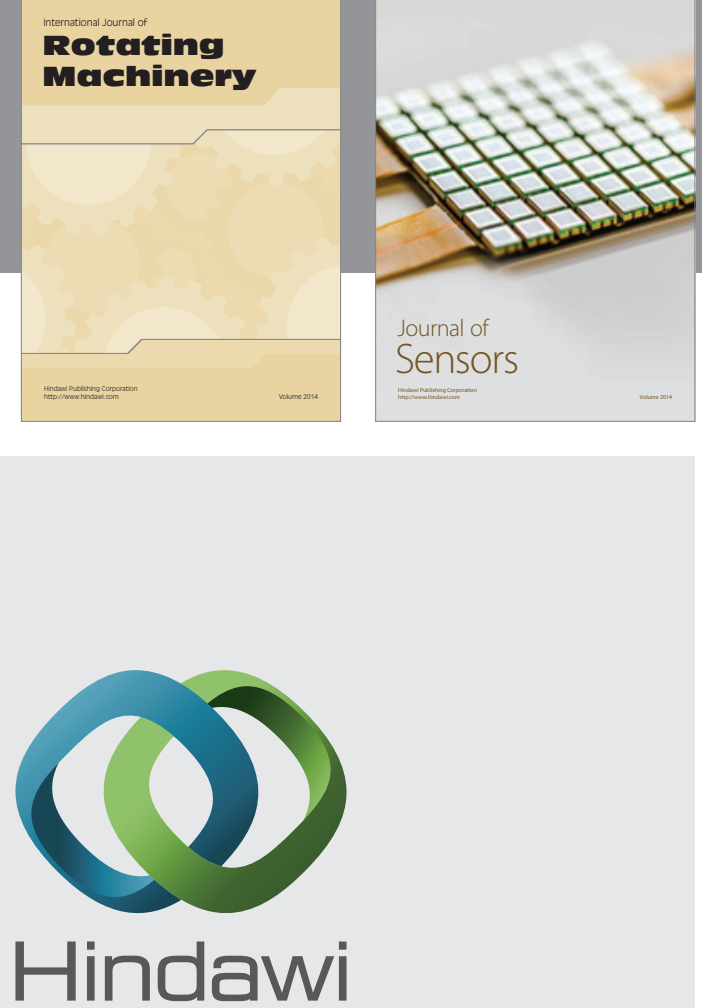

Submit your manuscripts at http://www.hindawi.com
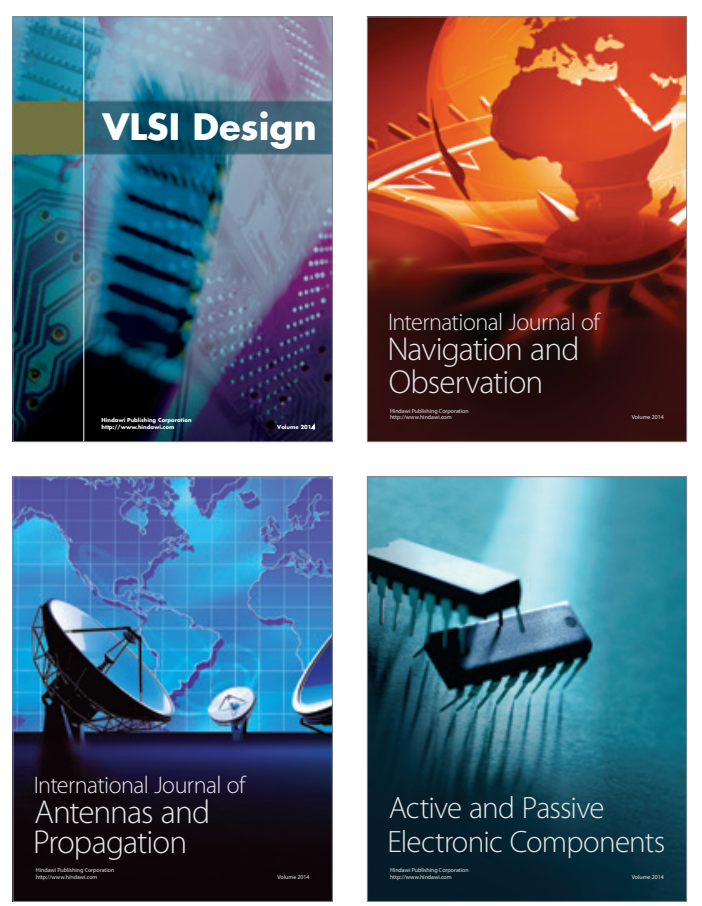
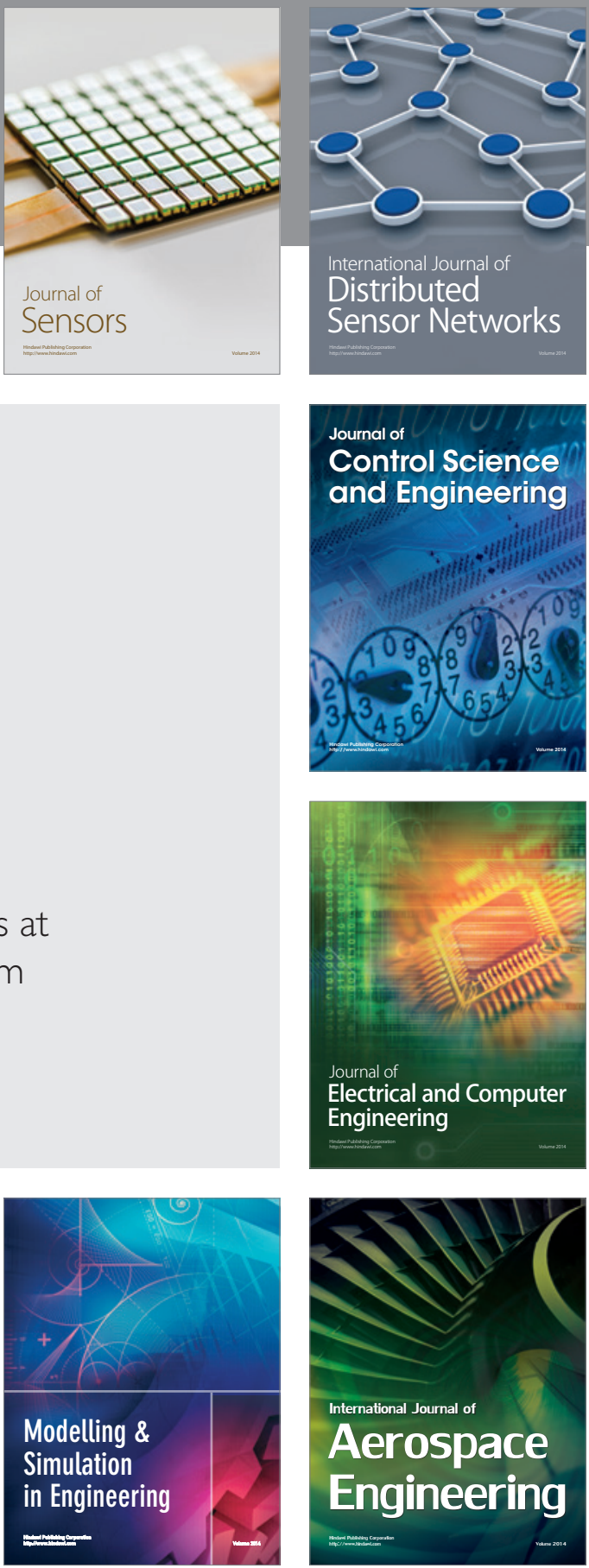

Journal of

Control Science

and Engineering
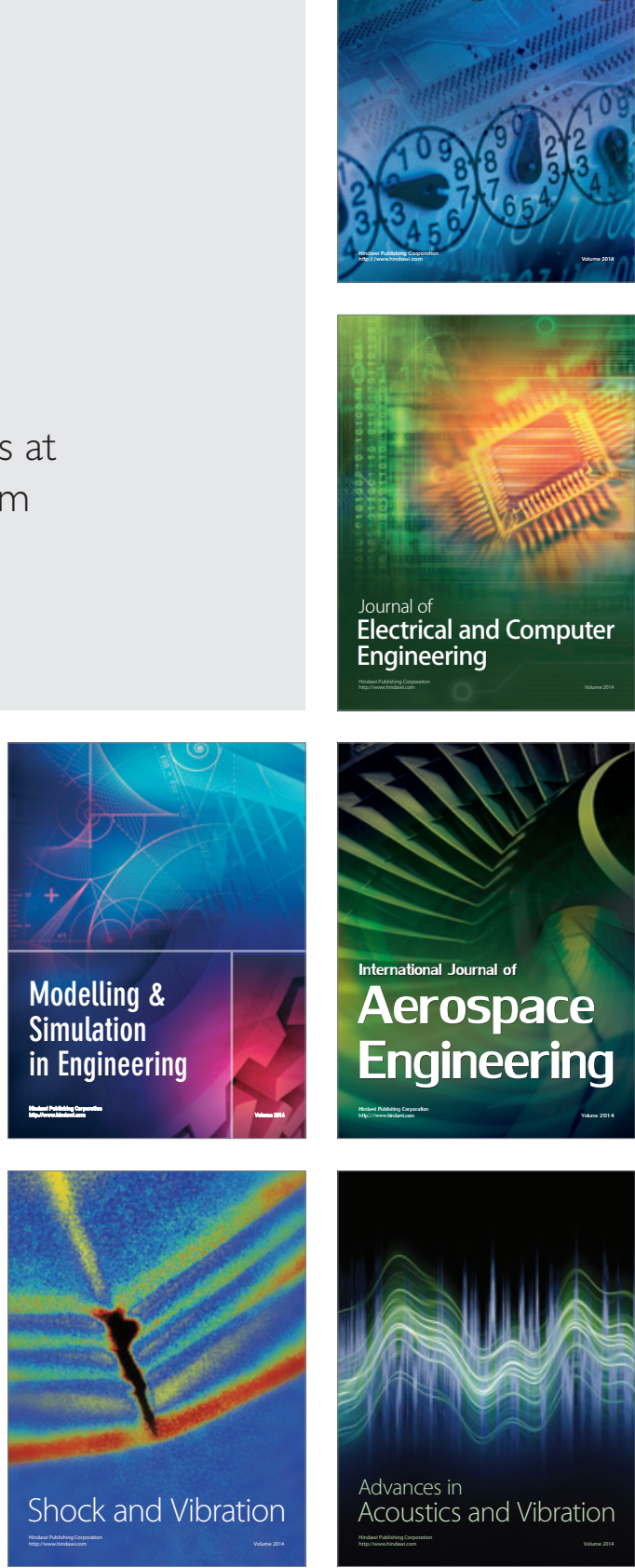